\title{
Functional anatomy of the levator palpebrae superioris muscle and its connective tissue system
}

\author{
A Ettl, S Priglinger, J Kramer, L Koornneef
}

Orbital Center,

Department of

Opthalmology,

Academic Medical

Center, Amsterdam, the Netherlands

A Ettl

L Koornneef

Department of

Ophthalmology,

General Hospital, St

Pölten, Austria

A Ettl

Institute for

Orthoptics, Hospital

Barmherzige Brüder,

Linz, Austria

$S$ Priglinger

CT and MRI Institute,

Linz, Austria

J Kramer

Correspondence to:

Dr med Armin Ettl,

Kaiserwaldweg 55 a, A-8010

Graz, Austria.

Accepted for publication

17 May 1996

\begin{abstract}
Aims/background-The connective tissue system of the levator palpebrae superioris muscle (LPS) consists of the septa surrounding its muscle sheath, the superior transverse ligament (STL) commonly referred to as 'Whitnall's ligament' and the common sheath which is the fascia between the LPS and the superior rectus muscle (SRM). The anterior band-like component of the common sheath is called transverse superior fascial expansion (TSFE) of the SRM and LPS. It mainly extends from the connective tissue of the trochlea to the fascia of the lacrimal gland. A detailed description of the relation between the LPS and its connective tissue is presented. Furthermore, the course of the LPS in the orbit is described. The study was conducted to provide a morphological basis for biomechanical and clinical considerations regarding ptosis surgery.
\end{abstract}

Methods-Postmortem dissections were performed in 16 orbits from eight cadavers. The microscopical anatomy was demonstrated in six formalin preserved orbits from six cadavers which had been sectioned in the frontal and sagittal plane and stained with haematoxylin and azophloxin. Surface coil magnetic resonance imaging in the sagittal and coronal plane was performed in five orbits from five normal volunteers using a T1 weighted spin echo sequence.

Results-The STL and the TSFE surround the LPS to form a fascial sleeve around the muscle which has attachments to the medial and lateral orbital wall. The TSFE, which is thicker than the STL, blends with Tenon's capsule. The STL and the fascial sheath of the LPS muscle are suspended from the orbital roof by a framework of radial connective tissue septa. MR images show that the TSFE is located between the anterior third of the superior rectus muscle and the segment of the LPS muscle where it changes its course from upwards to downwards. In this area, the LPS reaches its highest point in the orbit (culmination point). The culmination point is located a few millimetres posterior to the equator and superior to the globe.

Conclusion-Whitnall's ligament can be considered to consist of two distinct parts-the TSFE inferior to the LPS and the STL superior to the LPS. Since the medial and lateral main attachments of Whitnall's ligament are situated inferior to the level of the culmination point of the LPS, the ligament itself is unlikely to suspend the levator muscle. However, a suspension of the LPS may be achieved by the radial connective tissue septa of the superior orbit. The TSFE in connection with the globe may have an additional supporting function. The elasticity of Whitnall's ligament and its connections with highly elastic structures including Tenon's capsule, may provide the morphological substrate for the previously proposed passive (that is, without orbicularis action) lowering of the lid during downward saccades.

(Br F Ophthalmol 1996;80:702-707)

The preservation of the suspensory connective tissue system of the levator palpebrae superioris muscle (LPS) is regarded to be an important principle in ptosis surgery. ${ }^{1}$ According to the literature, the superior transverse ligament (STL) represents the main part of the suspensory system of the LPS. ${ }^{12}$

The superior transverse ligament (Whitnall's), is a condensation of the fascial sheath of the LPS on its superior surface which extends from the connective tissue complex of the trochlea medially, to the capsule of the orbital lobe of the lacrimal gland and the orbital wall laterally. The STL has also bilateral connections to the horns of the aponeurosis. The STL is largely located in the transitional zone between muscular levator and the aponeurosis. $^{3}$

In some patients with congenital ${ }^{2}$ and involutional ${ }^{4}$ ptosis, the STL has been observed to be atrophic or dehiscent. It has been suggested that these cases may benefit from repair of Whitnall's ligament in addition to conventional ptosis surgery. ${ }^{24}$

The function of the STL has been controversially discussed: Whitnall ${ }^{3}$ stated that the STL would act as a check ligament of the LPS. However, Lemke et $a l^{5}$ noted that the ligament is not under tension during lid closure and Dutton $^{6}$ believes that the check function of the STL is not significant under physiological conditions. Anderson and Dixon ${ }^{2}$ mentioned that larger amounts of levator resections are required if Whitnall's ligament is severed and therefore recommended its preservation during ptosis surgery. They suggested that the superior transverse ligament would act as a fulcrum which translates the anteroposterior force of the LPS into a vertical upward motion of the eyelid. Boergen and Scherz ${ }^{7}$ who cut 
Whitnall's ligament during large levator resections, stated that 'negative consequences' were not observed following this procedure.

The so called 'common sheath' is the intermuscular fascia between the LPS and the SRM. ${ }^{8}$ Fink ${ }^{9}$ has called its anterior part the 'transverse superior fascial expansion (TSFE) of the levator and superior rectus muscles'. Whitnall, ${ }^{3}$ Jones, ${ }^{8}$ and Dutton ${ }^{6}$ briefly mentioned the relation between the STL and the common intermuscular fascia.

The architecture of the connective tissue system of the orbit contributes to the course of the extraocular muscles and therefore ma have important functional implications. ${ }^{10}$ The present study was undertaken to investigate the course of the LPS muscle and its relation to the connective tissue system of the superior orbit. For this purpose, high resolution magnetic resonance imaging (MRI) was performed in vivo in addition to anatomical and histological studies. A series of photographs of macroscopic dissections is shown in order to illustrate the morphological relations for the eyelid surgeon.

\section{Material and methods}

Macroscopic anatomical dissections were performed in 16 orbits from eight unfixed cadavers (age range $40-85$ years) via a transconjunctival or a combined transcutaneous and transcranial approach.

En bloc excised and formalin fixed orbits from six cadavers (age range 26-73 years) which had been decalcified with ethylenediamine tetra-acetic acid, embedded in celloidin, serially sectioned $(60 \mu \mathrm{m})$ in the frontal plane $(n=3$ orbits) and in the sagittal plane $(n=3$ orbits), and stained with haematoxylin and azophloxin ${ }^{10}$ were analysed microscopically.

MRI of the orbit was performed in five volunteers (age range 29-54 years), after consent had been obtained, on a 1 Tesla scanner (Impact, Siemens, Germany) using a surface coil with a diameter of $10 \mathrm{~cm}$. Oblique sagittal (sections parallel to the optic nerve) and coronal (sections in the frontal plane) $\mathrm{T} 1$ weighted images of the orbit were obtained by spin echo sequences with an echo time (TE) of $15 \mathrm{~ms}$ and a repetition time (TR) of 440-520 ms. The slice thickness was $2-3 \mathrm{~mm}$ and there was no gap between slices. The field of view in the original images ranged between $140 \times 140$ $\mathrm{mm}$ with a $256 \times 256$ matrix and $230 \times 230$ $\mathrm{mm}$ with a $512 \times 512$ matrix. The acquisition time was between 2 and 13 minutes. Images were taken with both eyes closed (resting position in slight downgaze).

\section{Results}

ANATOMY

The STL and the TSFE unite at the medial and lateral margins of the LPS just proximal to the musculotendinous junction thus completely surrounding the LPS muscle (Fig 1). This fascial sleeve has attachments to the orbital walls medially and laterally. Medially, it joins the connective tissue of the trochlea and superior oblique muscle tendon (Fig 1). There are also extensions to the medial levator horn, the medial palpebral ligament, and check ligament of the medial rectus muscle. Laterally, there are weak attachments to the superolateral periorbit via the fascia of the lacrimal gland (Fig 2). More firm extensions insert into the lateral retinacular complex which includes the lateral palpebral ligament, the lateral check ligament, and the adjacent periorbit. The medial attachment of the fascial sleeve of the LPS is much thicker than the lateral attachment. Thin connective tissue septa pass in a more or less radial orientation from the STL through the preaponeurotic fat pad to the periorbit of the orbital roof and margin (Fig 3). The STL is connected to the LPS with stronger attachments at the medial and lateral borders of the muscle. Loose connective tissue connects the TSFE with the overlying LPS and the underlying SRM. Firm connections exist between the LPS and the SRM at their margins (Fig 4). The TSFE extends from the fascia of the lacrimal gland (Fig 2) towards the connective tissue of the superior oblique tendon and the trochlea (Fig 5). It starts at a level below the STL and extends posteriorly for about 10 $\mathrm{mm}$. The TSFE sends delicate connective tissue fibres into the superior fornix, previously described as the 'suspensory ligament of the superior fornix' (Fig 4).

If the LPS is reflected and the TSFE is carefully incised, the bare surface of the SRM and the sclera is exposed indicating that the TSFE represents a condensation of Tenon's capsule which blends with the fascial sheath of the muscles in this area (Fig 6).

Histological sections in the frontal plane (Fig 7) confirm that the STL and the TSFE unite at the medial and lateral borders of the LPS and extend further laterally to the capsule of the lacrimal gland and medially to the connective tissue of the trochlea. The TSFE blends with Tenon's capsule. Fibres from the TSFE course inferiorly to insert into the connective tissue of the medial and lateral rectus muscle. Posterior to the equator of the globe, the common sheath blends with the superolateral intermuscular septum and with Tenon's capsule medially. Throughout the length of the entire orbit, a network of radial septa connects the fascial sheath of the LPS with the periorbit of the orbital roof. Radial septa are also abundant in the region of the STL. The TSFE is considerably thicker than the STL. Sagittal sections demonstrated that the thickness of the fascia between the LPS and the SRM (common sheath) is continuously increasing from the posterior orbit towards the anterior orbit until it reaches its greatest thickness of about 2-3 mm in the area of the TSFE. Small amounts of adipose tissue are also noted in the space between the LPS and the SRM.

\section{MAGNETIC RESONANCE IMAGING}

On sagittal images (Fig 8), the LPS courses upwards from its origin until it reaches a culmination point (most cranial point) from where it courses downwards to the insertion in the upper lid. In resting position (closed lids, eye in slight down gaze), the culmination point is $14-16 \mathrm{~mm}$ posterior to the superior orbital 


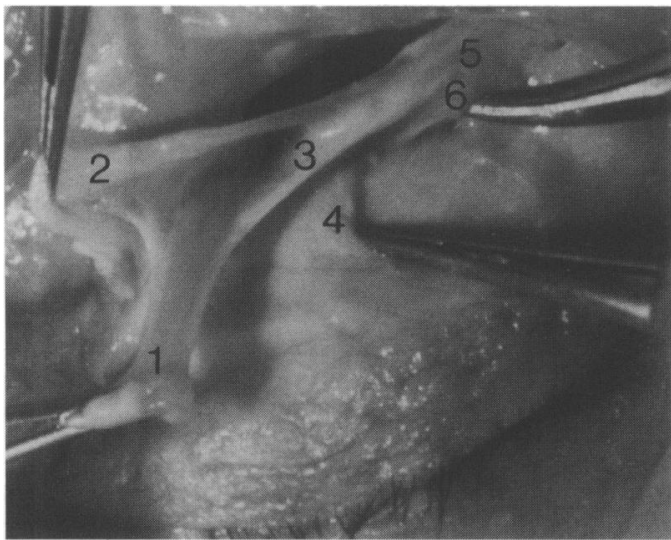

Figure 1 Anterior approach dissection (right upper lid). Following a lid crease incision, the levator palpebrae superioris (LPS) (1) has been cut anteriorly and pulled forwards. The superior transverse ligament (STL) (2) and the transverse superior fascial expansion (TSFE) (3) surround the LPS to form a fascial sleeve around the muscle. The superior rectus muscle (SRM) tendon (4) is under the TSFE. Medially, Whitnall's ligament inserts into the connective tissue complex (5) of the superior oblique tendon (6) and the trochlea.

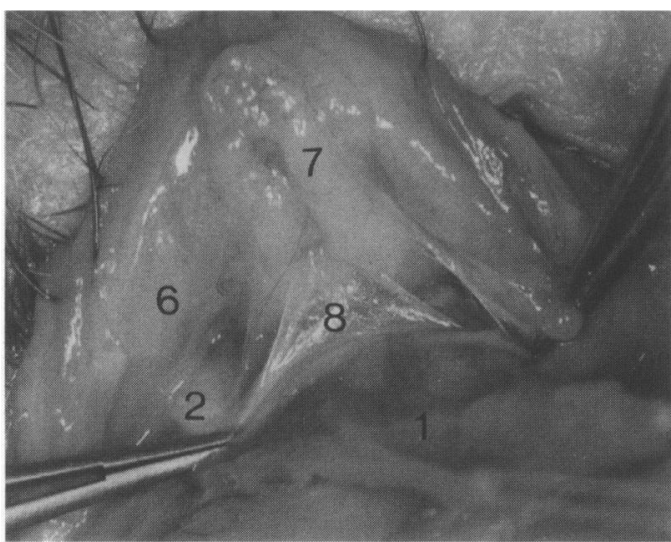

Figure 3 Posterior approach dissection (right upper lid). The orbital septum (6) has been reflected upwards and the levator muscle (1) has been pulled forwards. The preaponeurotic fat pad (7) has been elevated to show the radial septa (8) running from the STL (2) through the fat pad towards the orbital roof.

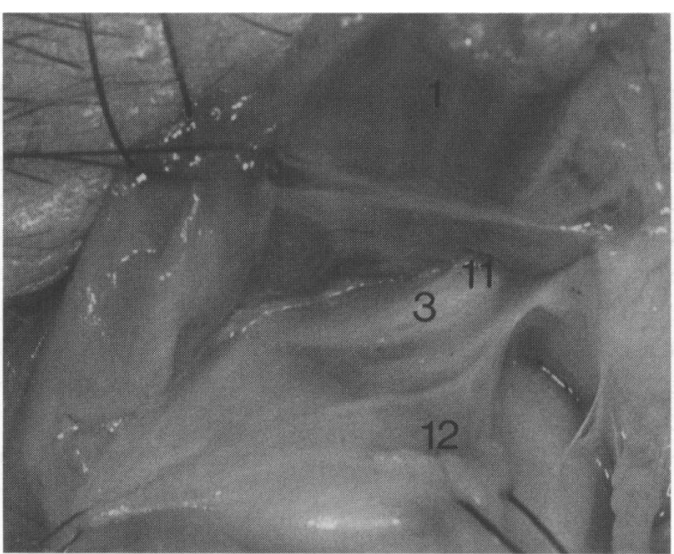

Figure 5 Posterior approach dissection (right upper lid). The connections between LPS (1) and SRM have been dissected off and the LPS has been reflected upwards to show the TSFE (3) extending from the lacrimal gland (not visible) towards the connective tissue complex (11) of the superior oblique tendon and the trochlea. Tenon's capsule (12) is overlying the insertion of the SRM.

margin and 5-7 $\mathrm{mm}$ posterior to the equator of the globe (horizontal distances). The culmination point is located $9-11 \mathrm{~mm}$ superior to the annulus tendineus and 4-5 $\mathrm{mm}$ superior to the

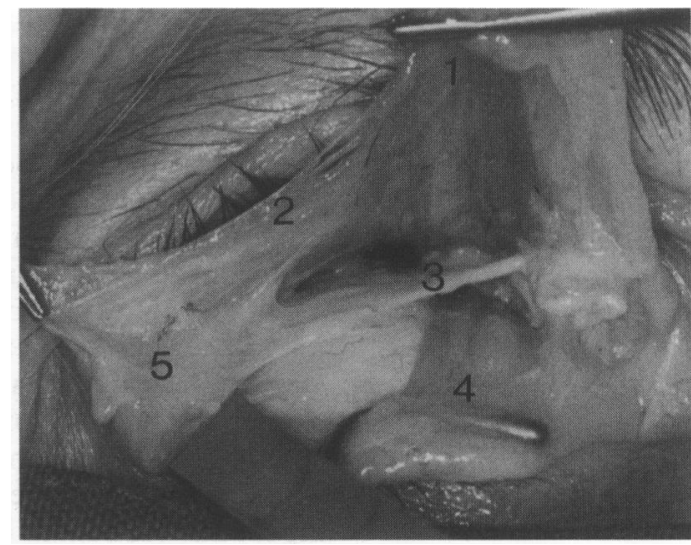

Figure 2 Posterior approach dissection (right upper lid). Following a conjunctival incision, the LPS (1) has been cut anteriorly and pulled upwards. Laterally the STL (2) and the TSFE (3) extend to the capsule of the lacrimal gland (5). SRM insertion (4).

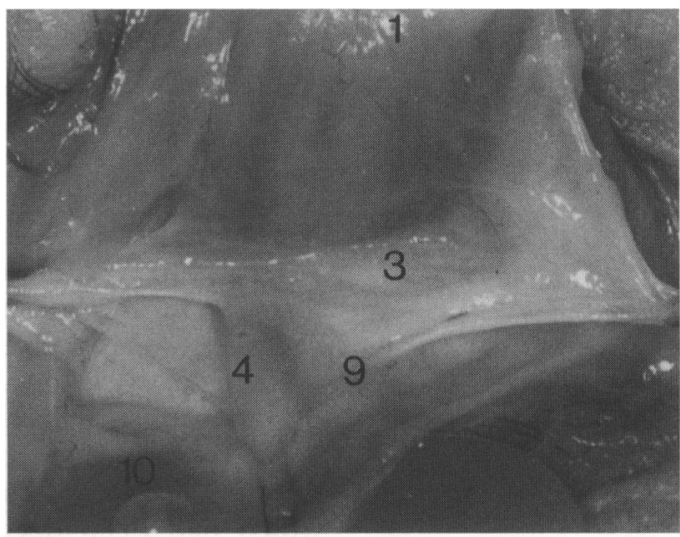

Figure 4 Posterior approach dissection (right upper lid). TSFE (3) between the LPS (1) and the SRM (4). The medial connections between SRM and LPS are thicker than the lateral connections. The suspensory ligament of the superior fornix (9) can be traced from the TSFE towards the conjunctival fornix (10) which is outlined with a piece of paper.

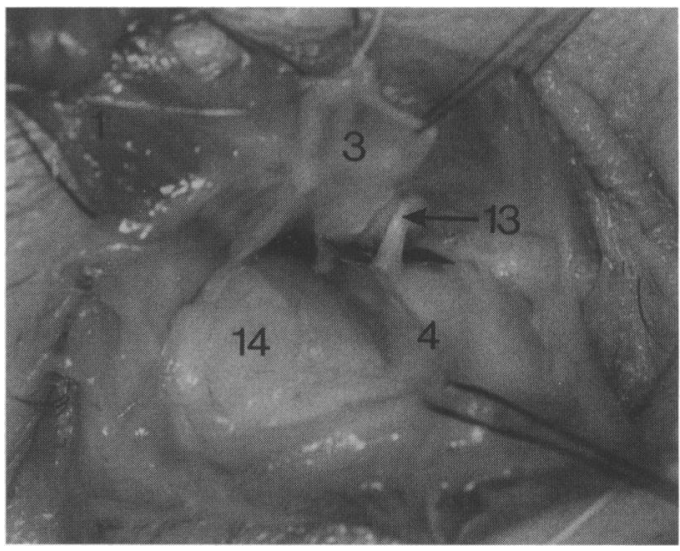

Figure 6 Posterior approach dissection (right upper lid). The TSFE (3) has been incised horizontally and reflected upwards: the reflected part of the superior oblique tendon (13), the SRM (4), and the bare surface of the sclera (14) are now exposed. No distinct separation between the TSFE and Tenon's capsule is found.

globe (vertical distances). The length of the levator aponeurosis between the upper tarsal border and the culmination point measures $22-25 \mathrm{~mm}$. The length of the LPS between its origin and the culmination point measures $36-40 \mathrm{~mm}$.

Fine septa are visualised between the upper part of the aponeurosis and the supraorbital 


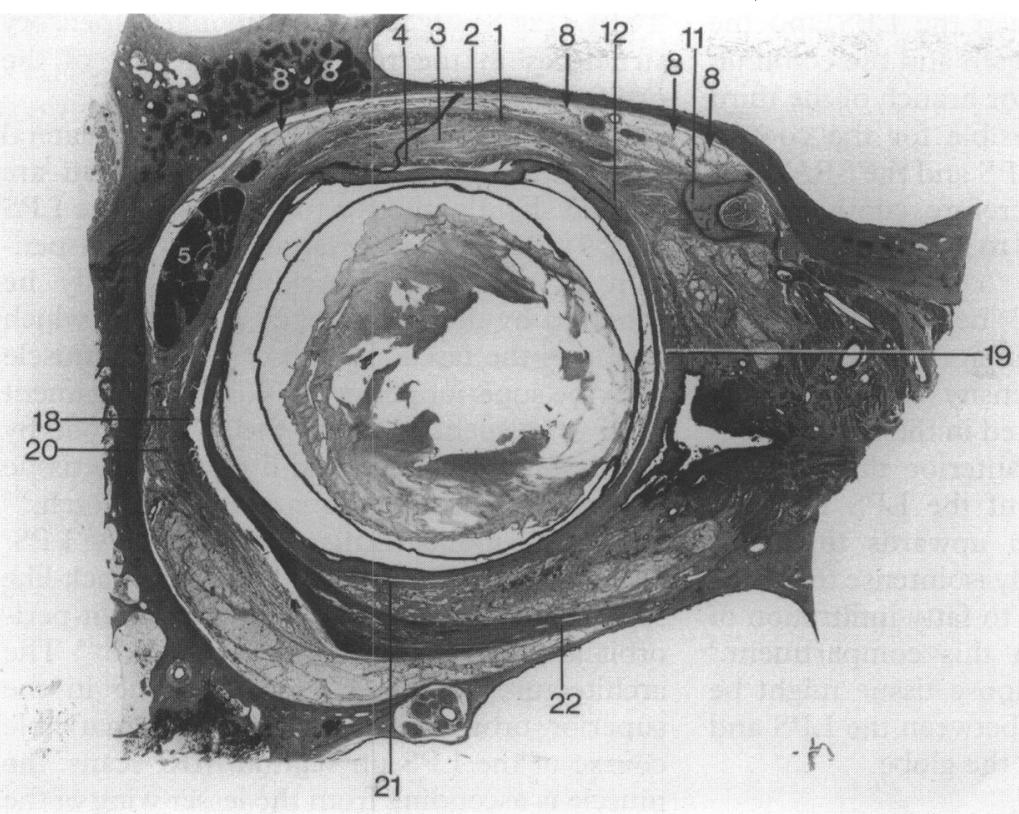

Figure 7 Histological section through a right orbit in the frontal plane at the level of the trochlea (11). The STL (2) and TSFE (3) blend at the borders of the LPS (1) and extend laterally to the capsule of the orbital lobe of the lacrimal gland (5) and medially to the connective tissue of the trochlea (11). At this level, the straight eye muscles are located within Tenon's capsule (12) and the TSFE blends with it. The TSFE is considerably thicker than the STL. Fibres of the TSFE extend to the connective tissue of the lateral (18) and medial rectus muscles (19). Radial septa (8) connect the STL with the superior periorbit. Lateral check ligament (20), inferior rectus muscle (21), inferior oblique muscle (22). Haematoxylin and azophloxin, original magnification $2.5 \times$.

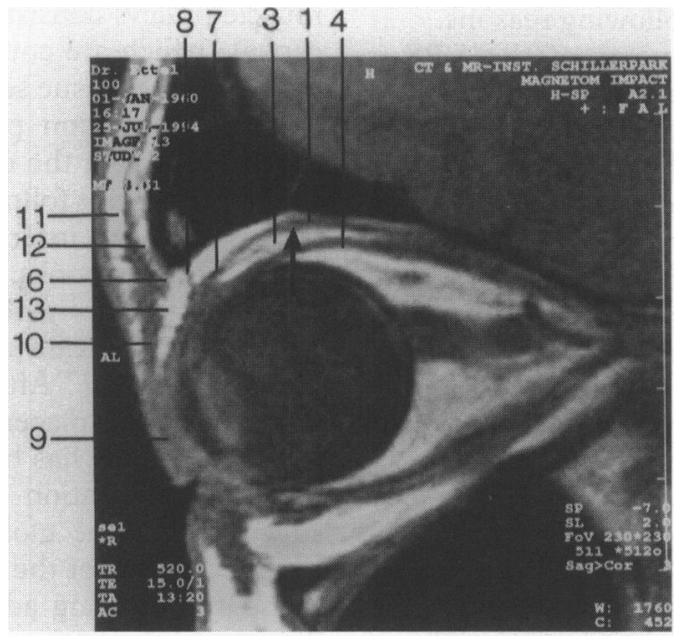

Figure 8 Sagittal MRI scan. The TSFE (3) is located in the space between the anterior LPS (1) and SRM (4). It is infiltrated with fatty tissue accounting for its isointensity to orbital fat. The hypointense structure inside this space is a connective tissue lamella. Posterior to the orbital septum (6), a short connective tissue septum (8) passes from the levator aponeurosis (7) through the preaponeurotic fat to the orbital roof. The LPS courses upwards from its origin to reach a culmination point (arrow) from where it courses downwards to the tarsal plate (9). The orbital septum joins the posterior surface of the orbicularis muscle (10) before uniting with the aponeurosis just above the superior tarsal border. Tissue compartments containing adipose tissue appear white in this $T 1$ weighted image. The subcutaneous fat (11) is visible between skin and orbicularis muscle. The brow fat pad (12) is noted between the orbicularis muscle and the orbital septum and the fat pad of the

preaponeurotic (postseptal) space (13) between orbital septum and aponeurosis. (Bar $=1 \mathrm{~cm}$.)

margin. The intermuscular space between the anterior third of the SRM and the segment of the LPS, where it changes its course from upwards to downwards, is isointense to orbital fat but also contains hypointense structures corresponding to parts of the TSFE and common sheath respectively.
On coronal slices through the equator of the globe, the TSFE is noted between SRM and LPS. The medial and lateral main attachments of Whitnall's ligament are visualised extending from the trochlea to the lacrimal gland and the lateral orbital wall (Fig 9).

\section{Discussion}

ANATOMY

The STL and the TSFE, form a fascial sleeve around the LPS which is attached to the medial and lateral orbital wall like an arc (Fig 10). Since the $\mathrm{TSFE}^{9}$ is connected to the STL and appears as a firm band-like structure, it has been referred to as the 'lower part of Whitnall's ligament' as opposed to the 'upper part of Whitnall's ligament' representing the STL (Priglinger S et al, Anatomie des Lig Whitnall und des oberen Muskelbindegewebsapparates der Orbita. Presented in 1991 at the 25th Strabismus Symposium of the Austrian Ophthalmological Society in St Pölten, Austria). Lukas

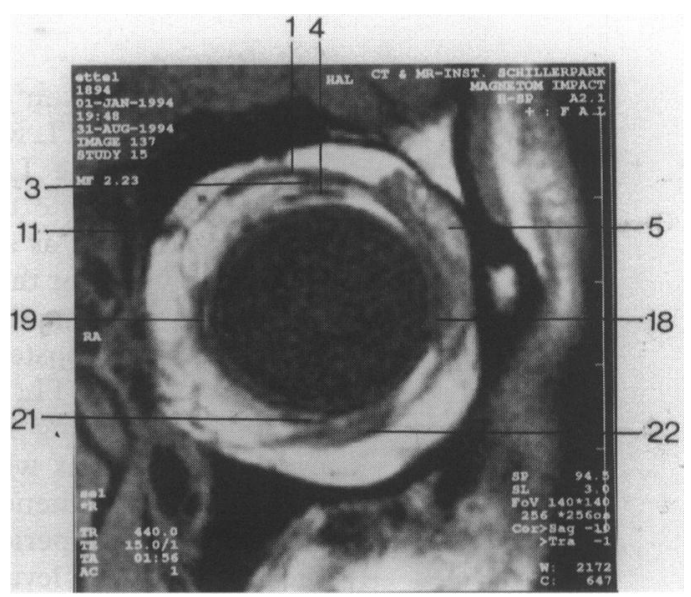

Figure 9 Coronal MRI scan of a left orbit at the level of the trochlea. The connections between the aponeurosis (1) and the SRM (4) belong to the TSFE (3). Extensions of Whitnall's ligament to the trochlea (11), to the check ligament of the medial rectus muscle (19) and to the lateral orbital wall and the orbital lobe of the lacrimal gland (5) are visible. Lateral expansions of the common sheath which blends with Tenon's capsule can be traced to the connective tissue of the lateral rectus muscle (18). Inferior rectus muscle (21), inferior oblique muscle (22).

et $a l^{112}$ confirmed that the connective tissue underlying the anterior portion of the LPS has the characteristic anatomical and histological features of a ligament and proposed the name 'transverse intermuscular ligament' (ligamentum transversum intermusculare). The attachments of Whitnall's ligament to the orbital wall and the interconnecting fibres between the TSFE and the LPS are more strongly developed medially than laterally whereas the lateral horn of the aponeurosis is much stronger than the more elastic medial horn. Coronal MRI scans confirm these findings. This configuration may contribute to the normal lid contour which has its peak slightly medial to the centre of the pupil.

The LPS can glide within the sling formed by Whitnall's ligament only to a small extent, due to fibroelastic connections between muscle and ligament. ${ }^{12}$ Therefore the ligament must follow the excursions of the LPS which was concluded from a previous MRI study. ${ }^{13}$ 
The connection between the LPS and the SRM by the common sheath and the common innervation by the superior branch of the third cranial nerve are responsible for the coordinated movement of the LPS and the SRM during vertical saccades. Therefore, contraction of the SRM accounts for up to $2 \mathrm{~mm}$ of the entire upper lid elevation. ${ }^{?}$

The STL could not be identified with certainty in our MR images owing to its thinness and the isointensity to aponeurotic tissue. The TSFE is located in the intermuscular space between the anterior third of the SRM and the segment of the LPS where it changes its course from upwards to downwards. This space is largely isointense to orbital fat on MRI which is due to fatty infiltration of the connective tissue in this compartment. ${ }^{5}$ The function of this adipose tissue might be the reduction of friction between the LPS and the underlying SRM and the globe.

\section{FUNCTIONAL CONSIDERATIONS}

\section{Lid elevation}

Anderson and Dixon ${ }^{2}$ and Goldberg et $a l^{13}$ suggested that the STL would act as a fulcrum or suspender for the LPS. However, our MR images demonstrate that the STL alone may not be able to act as a suspensory ligament of the LPS muscle for the following reasons.

(1) The culmination point of the LPS is situated slightly posterior and superior to the location of the STL. This has recently been demonstrated using high resolution MRI in cadaver specimens where the STL had been marked with synthetic material (Ettl A, et al, Relation of the superior transverse ligament to the course of the levator palpebrae superioris muscle, unpublished observation, 1995).

(2) The culmination point of the LPS is located superior to the posterior part of the

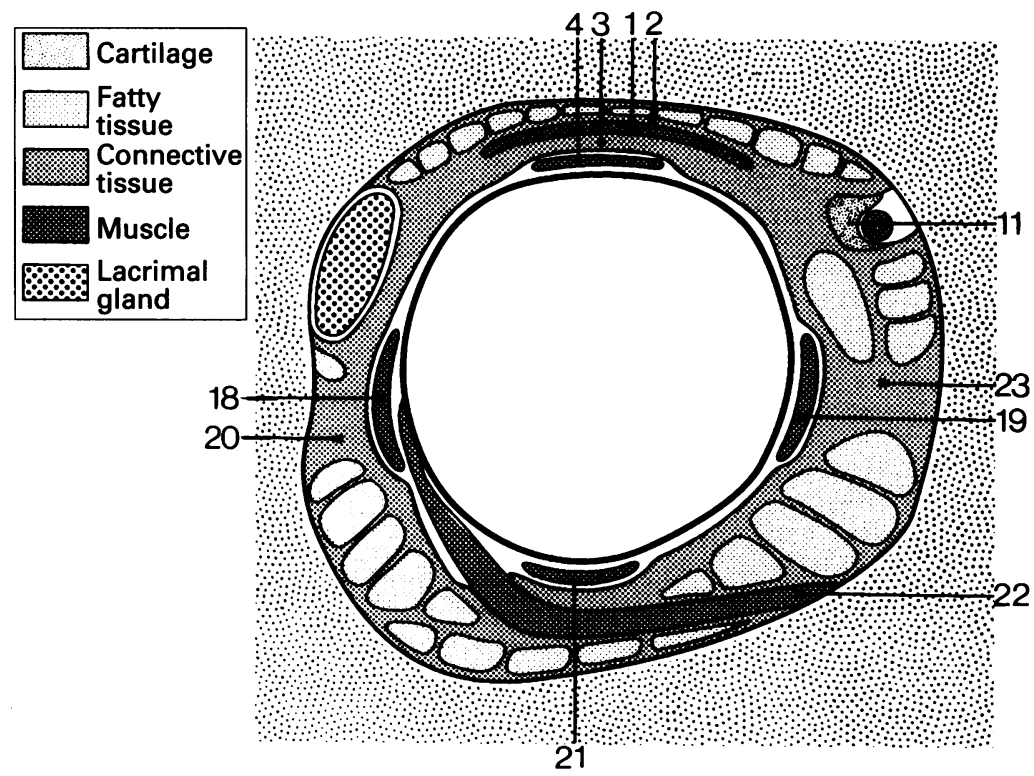

Figure 10 Diagrammatic representation of the connective tissue system in the anterior orbit: Whitnall's ligament $(2,3)$ completely surrounds the LPS muscle (1). The nomenclature is explained in the legend to Figure 9. Medial check ligament (23). (Schematic synthesis of two frontal sections through the trochlea and just posterior to the trochlea.)
TSFE (Fig 8) suggesting additional suspensory structures in the retroequatorial part of the orbit.

(3) The superomedial and the superolateral main attachments of Whitnall's ligament are located slightly inferior to the level of the LPS (Fig 9). Therefore, we argue that the suspension of the LPS muscle may actually be achieved by the radial septal system ${ }^{10}{ }^{14}$ which connects the fascial sheath of the LPS muscle with the superior periorbit. Whitnall's ligament itself is suspended from the orbital roof by means of vertical septa and connective tissue strands coursing to the supraorbital notch. ${ }^{36}$ Behind the globe, further support for the LPS/ SRM complex is provided by hammock-like septa which are anchored to the superior periorbit at the margins of the muscles. ${ }^{1014}$ The architecture of the connective tissue in the superior orbit could explain the remarkable course of the LPS on sagittal MRI scans: the muscle is ascending from the lesser wing of the sphenoid to a culmination point several millimetres behind the equator and above the globe from where the aponeurosis is descending to the insertion ${ }^{15}$ in the eyelid (Fig 8). The deflection of the LPS leads to a lengthening of the muscle path which may increase the muscle tension due to increased stretch of the muscle. This function is comparable with the rectus muscle pulleys consisting of sleeves in Tenon's capsule which are coupled to the orbital walls by connective tissue septa. ${ }^{16}$

The culmination point of the LPS is not exactly overlying the equator of the globe and the LPS does not follow the shortest path from the origin to the insertion as often depicted in anatomical textbooks. Such a course would be expected if the globe alone provided the fulcrum for the LPS as suggested by Vistnes ${ }^{17}$ and Lemke et $a .^{5}$ After removal of the eye, a downwards displacement of the superior muscle complex has been described as part of the post enucleation socket syndrome. ${ }^{18}$ This suggests that the globe obviously prevents a partial collapse of the ocular motion compartment by providing additional support for the TSFE and the LPS.

\section{Lid lowering}

We have demonstrated that the TSFE blends with Tenon's capsule. The connections of Whitnall's ligament with tissue structures containing elastic fibres or smooth muscle fibres such as Tenon's capsule, intermuscular septum, ${ }^{1014}$ radial septa, ${ }^{1014}$ and aponeurosis ${ }^{19}$ are responsible for the relatively high elasticity of the upper lid. Furthermore, both parts of Whitnall's ligament contain elastic fibres which are especially abundant in the connections between the STL and the LPS muscle and the TSFE and the SRM allowing for a small amount of movement of the LPS in the sling formed by Whitnall's ligament. ${ }^{12}$

Loss of elasticity of the suspensory connective tissue system of the eyelid explains the lid lag observed following levator resections ${ }^{1}$ and in patients suffering from Graves' disease. ${ }^{20}$

The above described fibroelastic attachments may prevent abrupt stops of the lid 
movements at extreme upgaze and downgaze. In downgaze, the central portion of Whitnall's ligament moves further anteriorly than the medial and lateral attachments producing a bow-shaped configuration of both parts of the stretched ligament so that its convexity is anteriorly directed. Whitnall's ligament may therefore suspend the upper eyelid (but not the LPS muscle) in downgaze.

Based on electromyography and the magnetic search coil technique, it has been suggested that the elastic forces of the eyelid connective tissue system are responsible for the motion pattern of the lid during downward saccades. ${ }^{21}$ According to this hypothesis, the LPS must stretch the 'connective tissue spring' when elevating the eyelid. Relaxation of the LPS releases the energy stored in the stretched connective tissue and causes a rapid lowering of the eyelid.

The present study has described some morphological and radiological details regarding the LPS muscle and its connective tissue system. Further biomechanical considerations and surgical applications are the subject of our ongoing research.

Note added on proof:

Similar anatomical findings were published by other authors ${ }^{22}$ after submission of our study.

The authors thank Professor F Zonneveld (Department of Radiology, University Hospital Utrecht, the Netherlands) for valuable discussions.

Dortzbach RK. Ophthalmic plastic surgery. New York: Raven 1994:68-9.

2 Anderson RL, Dixon RS. The role of Whitnall's ligament in ptosis surgery. Arch Ophthalmol 1979;97:705-7.
3 Whitnall SE. On a ligament acting as a check to the action of the levator palpebrae superioris muscle. $\mathcal{F}$ Anat Physiol 1910;45:131-9.

4 Shore JW, McCord CD. Anatomic changes in involutional blepharoptosis. Am $\mathcal{f}$ Ophthalmol 1984;98:21-7.

5 Lemke BN, Stasior OG, Rosenberg PN. The surgical relations of the levator palpebrae superioris muscle. Ophthalmic Plast Reconstr Surg 1988;4:25-30.

6 Dutton J. Atlas of clinical and surgical orbital anatomy. Philadelphia: Saunders, 1994:96-7.

7 Boergen KP, Scherz W. Prosis. Munich: Quintessenz, 1994:17-58.

8 Jones LT. A new concept of the orbital fascia and rectus muscle sheaths and its surgical implications. Trans Am Acad Ophthalmol Otolaryngol 1968;72:755-64.

9 Fink WH. An anatomic study of the check mechanism of the vertical muscles of the eye. Am f Ophthalmol 1957;44 800-9.

10 Koornneef L. Spatial aspects of musculo-fibrous tissue in man. Lisse: Swets \& Zeitlinger, 1976:1-168.

11 Lukas JR, Denk M, Mayr R. Connective tissues related to the levator palpebrae superioris muscle-an anatomical study on 120 human orbitae. Ann Anat 1994;176(suppl): 97-8.

12 Lukas JR, Denk M, Mayr R. The anatomy, histology and function of an intermuscular transverse band underlying the levator palpebrae

13 Goldberg RA, Wu JC, Jesmanowicz A, Hyde JS. Eyelid anatomy revisited. Dynamic high-resolution magnetic resonance images of Whitnall's ligament and upper eyelid structures with the use of a surface coil. Arch Ophthalmo 1992;110:1598-600.

14 Koornneef $\mathrm{L}$. New insights in the human orbital connective tissue. Arch Ophthalmol 1977;95:1269-73.

15 Collin JRO, Beard C, Wood I. Experimental and clinical data on the insertion of the levator palpebrae superioris data on the insertion of the levator palpe
muscle. Am $¥$ Ophthalmol 1978;85:792-801.

16 Demer JL, Miller JM, Poukens V, Vinters HV, Glasgow BJ. Evidence for fibromuscular pulleys of the recti extraocular Evidence for fibromuscular pulleys of the recti extrao
muscles. Invest Ophthalmol Vis Sci 1995;36:1125-36.

17 Vistnes LM. Mechanisms of upper lid ptosis in the anophthalmic orbit. Plast Reconstr Surg 1976;58:539-45.

18 Smit TJ, Koornneef L, Zonnefeld FW, Groet E, Otto AJ Computed tomography in the assessment of the postenucleation socket syndrome. Ophthalmology 1990;97: 1347-51.

19 Stasior GO, Lemke BN, Wallow IH, Dortzbach RK. Levator aponeurosis elastic fiber network. Ophthalmic Plast Reconstr Surg 1993;9:1-10.

20 Koornneef L. Eyelid and orbital fascial attachments and their clinical significance. Eye 1988;2:130-4.

21 Evinger C, Manning K, Sibony PA. Eyelid movements. Evinger $\mathrm{C}$, Manning $\mathrm{K}$, Sibony PA. Eyelid movements.
Mechanisms and normal data. Invest Ophthalmol Vis Sci 1991;32:387-400.

22 Codere F, Tucker NA, Renaldi B. The anatomy of Whitnal ligament. Ophthalmology 1995;102:2076-9. 